\title{
Spatial vs. temporal controls over soil fungal community similarity at continental and global scales
}

\author{
Colin Averill $^{1,2} \cdot$ LeAnna L. Cates ${ }^{3} \cdot$ Michael C. Dietze $^{2} \cdot$ Jennifer M. Bhatnagar ${ }^{1}$
}

Received: 24 October 2018 / Revised: 21 February 2019 / Accepted: 20 March 2019 / Published online: 24 April 2019

(c) International Society for Microbial Ecology 2019

\begin{abstract}
Large-scale environmental sequencing efforts have transformed our understanding of the spatial controls over soil microbial community composition and turnover. Yet, our knowledge of temporal controls is comparatively limited. This is a major uncertainty in microbial ecology, as there is increasing evidence that microbial community composition is important for predicting microbial community function in the future. Here, we use continental- and global-scale soil fungal community surveys, focused within northern temperate latitudes, to estimate the relative contribution of time and space to soil fungal community turnover. We detected large intra-annual temporal differences in soil fungal community similarity, where fungal communities differed most among seasons, equivalent to the community turnover observed over thousands of kilometers in space. inter-annual community turnover was comparatively smaller than intra-annual turnover. Certain environmental covariates, particularly climate covariates, explained some spatial-temporal effects, though it is unlikely the same mechanisms drive spatial vs. temporal turnover. However, these commonly measured environmental covariates could not fully explain relationships between space, time and community composition. These baseline estimates of fungal community turnover in time provide a starting point to estimate the potential duration of legacies in microbial community composition and function.
\end{abstract}

\section{Introduction}

Soil fungi mediate a multitude of ecosystem functions with major Earth system consequences, including primary production, nutrient recycling, and carbon (C) sequestration in soils [1]. Fungal communities vary in both space and time, due to changes in environmental conditions, ecological interactions, and neutral ecological and evolutionary processes [2, 3]. Quantifying the relative

Supplementary information The online version of this article (https:// doi.org/10.1038/s41396-019-0420-1) contains supplementary material, which is available to authorized users.

Colin Averill

caverill@bu.edu

1 Department of Biology, Boston University, 5 Cummington Mall, Boston, MA, USA

2 Department of Earth \& Environment, Boston University, Boston, MA, USA

3 Department of Biology, University of Missouri-Kansas City, Kansas City, MO, USA importance of spatial and temporal controls over community turnover is critical to understanding which of these processes drive community change, as fundamentally different ecological mechanisms likely control microbial turnover in space and time. For instance, while dispersal and colonization traits may fundamentally constrain species turnover in space $[4,5]$, dormancy and persistence traits may be more linked to species turnover in time [6]. Furthermore, fungal species vary in their ability to take up soil resources (e.g., $\mathrm{C}$, nitrogen $(\mathrm{N})$, and moisture) and in their tolerance for soil chemistry $(\mathrm{pH})$ [7-9], such that variation in these environmental factors across space and time may further shape fungal community composition in soil. In biogeographic analyses, changes in soil microbial community composition are largely attributed to space [10-12]. However, the handful of studies that have remeasured microbial communities through time often find large temporal variability in microbial community composition [13]. Given that most biogeographic analyses of microbial community composition are based on samples taken over multiple years [12], it may be that that some additional amount of unexplained variation can be attributed to time (Fig. 1). 


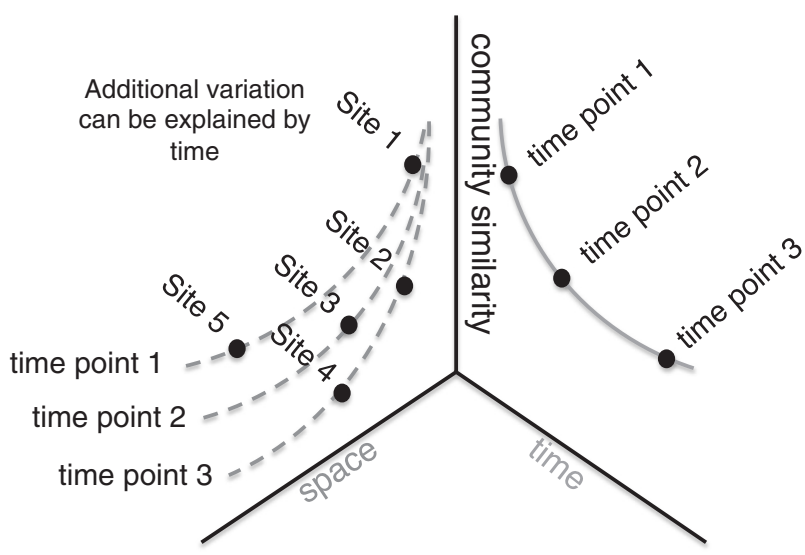

Fig. 1 Multiple sites in space are sampled at different points in time. Additional variation in spatial turnover can be attributed to underlying temporal processes

Studies of soil fungal community turnover in time are remarkably few, and local in spatial scale. These studies generally observe significant turnover in soil fungal communities in time [14, 15], where intra- and inter-annual turnover is comparable in magnitude to within site spatial turnover. However, it is well-known that soil microbial communities can differ wildly at scales of a meter or less [16], and therefore some have argued that at larger spatial scales soil microbial turnover will be dominated by spatial, rather than temporal dynamics $[17,18]$. Testing this assumption would require analyzing the relative importance of spatial vs. temporal drivers of soil community turnover across a large spatial scale.

To date, no large-scale spatial sampling of microbial community composition has been temporally replicated, so analyses of orthogonal observations of microbial community composition in space and time are not yet possible. In these studies, there is no location in space that has been sampled at multiple instances in time, primarily due to logistical constraints. It is fundamentally challenging to revisit a large, spatially distributed set of sites multiple times, and as a result, these surveys are often conducted over multiple years. However, the variation in the time of sampling is also an opportunity to quantify the relative importance of space and time in structuring microbial communities. If spatial and temporal distances are not completely confounded, it may be possible to separate the independent effects of space and time on community similarity using microbial community surveys currently available (Fig. 1).

Understanding temporal controls over microbial community composition is increasingly important in the context of global change. There have been repeated demonstrations of microbial functional resistance in the face of environmental manipulation [19], such that predicting microbial community function requires understanding not only current, but historical environmental conditions (i.e., "legacy" effects) [20-23]. If observed legacy effects on microbial community function are driven by resistance of microbial community composition to environmental change, then the duration of functional legacies will depend on the rate of microbial community turnover in time. Currently, there is little constraint on our estimates of the baseline temporal rate of microbial community turnover that could help us understand the potential duration of microbial functional legacies with any generality.

Here, we quantify individual contributions of geographical space and time to community composition, as well as the role of environmental factors in each relationship. We hypothesized that community turnover over several years may be equal in magnitude to that observed over hundreds of kilometers, as many important environmental factors (i.e., weather, climate, and soil resources) exhibit large variation between years. Furthermore, we hypothesized that community turnover may be faster within than between years, as the variation in climate and soil resources due to intra-annual seasonality is generally larger than inter-annual changes in climate [24]. In addition, intra-annual changes in soil resources for fungi (i.e., $\mathrm{C}, \mathrm{N}$, and water) may play a larger role in controlling community composition than climate, since fungi respond strongly to changes in resource availability [8]. To test these hypotheses, we first tested if known spatial and temporal effect sizes can be captured in the absence of repeated temporal sampling. Specifically, we quantified spatial and temporal community turnover in previously published studies of local forest, soil fungal and soil bacterial communities that had been sampled repeatedly in both space and time. After demonstrating this property, we analyzed variation in soil fungal community composition over space and time in two large datasets: a continental-scale and a global-scale sampling of temperate latitude forest soil fungal communities. We focused on northern temperate latitudes so that we can capture both intra- and inter-annual temporal variation in soil fungal community turnover among sites with a distinct growing season. We then asked: (1) what is the relative importance of space vs. time (both within and between years) in predicting community similarity in soil microbial communities at large spatial scales?; (2) how are rates of turnover in time and space related?; and (3) can spatial-temporal community turnover be explained by variation in environmental factors?

\section{Methods}

\section{Analysis overview}

In each data set, we analyzed pairwise community similarity using the Bray-Curtis metric [25] as a function of 
space and time. We analyze relative abundances of fungal taxa, because both presence/absence and changes in relative abundance of taxa are important for understanding the structure and function of ecological communities [26]. Because of potential intra-annual seasonal cycles in temperate latitude soil fungal community similarity, we fit community similarity to both intra-annual and inter-annual temporal distances. Significant relationships of each predictor to fungal community composition were determined using multiple regression analysis on distance matrices (MRM). To calculate $95 \%$ confidence intervals of each parameter estimate, we performed 10,000 bootstrap simulations. Initial diagnostic plots were used to decide whether or not to $\log$ transform Bray-Curtis metrics prior to analysis. Bray-Curtis scores were modeled as a linear function of space, time, and appropriate covariates. For each data set we fit three models; (1) a model that only included spatial and temporal predictors, (2) a model that only included environmental predictors, and (3) a model that included both spatial-temporal and environmental predictors. Comparing outputs of these sets of models allowed us to understand how fungal communities vary in space and time, as well as the degree to which these spatial-temporal relationships can or cannot be explained by variation in commonly measured environmental factors. Below we describe how species matrices were defined for each data set.

\section{Is orthogonal spatial and temporal sampling required to capture temporal patterns?}

Most analyses of microbial community similarity through time are based on repeated observations of communities through time at multiple fixed points in space [13], allowing quantification of both spatial and temporal effects simultaneously. While this type of sampling is ideal, it is rare to have observations collected in this way. In most observational studies, there is no location that has been sampled at multiple instances in time, and no single instance in time sampled at multiple positions in space. However, if space and time are not completely confounded, it should be possible to use this information to estimate the independent effects of space and time on community similarity without the need for repeated measures. We assess the validity of this approach using four previously published data sets that have observed community composition at the same location multiple times (i.e., observations of space and time are orthogonal). The first data set contains tropical tree community composition data from Barro Colorado Island, Panama (BCI) sampled 8 times over 35 years [27-29]. The second and third data sets contains soil bacterial and fungal community composition from La Selva Research Station in Costa Rica, sampled 4 times over 2 years [30]. The fourth data set was contains soil fungal community composition from the Harvard Forest National Ecological Observation Network (NEON) site, sampled 4 times during the 2014 growing season. We first analyze all data to quantify "true" spatial and temporal effects. We then subset these data sets such that no location has been sampled at multiple time points. We then refit models to determine if the detected effects of both space and time in the data subsets reflect the 'true' effects detected in the full data sets. Histograms of spatial and temporal distances within each study site are presented in Supplementary Fig. 1.

\section{$\mathrm{BCl}$ analysis}

The 50 ha BCI permanent tree plot, located in Gatun, Panama, has been censused 8 times between 1982 and 2015, at approximately 5-year intervals, with every tree in the plot identified and its spatial position recorded [27-29]. To determine if temporal signals in community similarity can be inferred from non-orthogonal spatial-temporal community data, we binned the plot into $300,40 \times 41.67 \mathrm{~m}$ $\left(\sim 1667 \mathrm{~m}^{2}\right)$ grid cells. Within each cell, the number of trees by species was tallied to generate community matrices for all eight time points. Bray-Curtis similarity among plots was calculated using proportionally normalized community matrices, and modeled as a linear function of spatial distance (using mean plot center based on the positions of all trees within the plot) and temporal distance (using mean date of sampling of all trees within a grid cell for a given census period). We then repeated the analysis, sub-setting the data randomly 100 times such that no particular plot had been observed at more than one time point, generating nonorthogonal space-time observations. We compared effects of both space and time in each analysis and checked if the 95\% confidence intervals of estimated parameter values overlap.

\section{La Selva bacteria and fungi analyses}

Bacterial and fungal community analysis was conducted on an operational taxonomic unit (OTU) tables from the same tropical forest sequencing experiment [30, 31]. Soil samples were collected from La Selva Biological Station in Costa Rica, under 25-year-old monodominant stands of four tree species: Hyeronima alchorneoides, Pentaclethra macroloba, Virola koschnyi, and Vochysia guatemalensis [32]. Samples of bacterial and fungal communities used in this study were taken from four replicate blocks of each stand using a $2.5 \mathrm{~cm}$ diameter soil corer to a depth of $10 \mathrm{~cm}$ [30]. Sites were sampled 4 times in March and September of 2012 and 2013. Soil cores were sieved, and subsamples were extracted using a MoBio PowerSoil Kit (MoBio, Carlsbad, CA). The 16S bacterial rDNA region 
and D1/D2 variable fungal region were amplified and then $2 \times 300$ paired end sequenced on an Illumina Miseq sequencer using v3 chemistry. Sequences were clustered into OTUs at $97 \%$ similarity using the UCLUST algorithm. Singletons, and OTUs found in only a single sample, were discarded. While removing singletons is common practice [33], removing OTUs found only within a single sample is less common. However, in principle this should decrease the statistical power of this data set, and make this analysis more conservative, as there is less information to distinguish among samples. Taxonomy was assigned using the Ribosomal Database Project (RDP) classifier, and nonbacterial OTUs discarded [34]. Samples were then normalized with the DeSeq2 algorithm, which leverages variation in sequencing depth among samples to account for overdispersion and heteroscedasticity in microbiome count data [35]. The original authors provided the processed OTU tables and full sampling and bioinformatic details can be found in ref. [30]. We proportionally normalized OTU tables to calculate Bray-Curtis community similarity among soil samples using the vegdist function within the vegan package for $\mathrm{R}$ statistical software [36, 37]. Sampling dates were used to calculate temporal distance in days. Spatial distances in kilometers were calculated based on $x-y$ coordinates of sampling locations reported by authors. We then repeated the analysis, sub-setting the data randomly 100 times such that no particular plot had been observed at more than one time point, generating nonorthogonal space-time observations. We compared effects of both space and time in each analysis and checked if the $95 \%$ confidence intervals of estimated parameter values overlapped.

\section{Harvard Forest fungal analysis}

Harvard Forest fungal sequence data were obtained from a preliminary sampling effort by the National Ecological Observation Network. Briefly, 2-6 soil samples were collected from random positions within $11,40 \times 40 \mathrm{~m}$ plots 4 times through the 2014 growing season (May, June, July, and August samplings). DNA was extracted using a MoBio PowerSoil Kit (MoBio, Carlsbad, CA). The fungal rDNA region internal transcribed spacer 1 (ITS1) was polymerase chain reaction (PCR) amplified and then $2 \times 250$ paired end sequenced on an Illumina Miseq sequencer with v2 chemistry. Reverse reads were discarded, and reads were quality filtered using default parameter settings in the QIIME pipeline [38]. Quality filtered reads were then dereplicated to construct amplicon sequence variant tables [39]. Chimeras were removed using dada 2 and singletons discarded [40]. Similarity matrix construction, analysis, subsetting, and bootstrapping were done as above for La Selva bacteria and fungi.

\section{Estimating temporal effect sizes from large-scale soil fungal community data}

\section{Talbot et al. [11]—North American Soil Fungi}

This dataset includes 550 observations of soil fungal community composition from North American pine forests collected over 728 days with a standardized sampling methodology [11]. DNA was extracted from $10-\mathrm{cm}$ soil cores using the same PowerMax Soil DNA Isolation kit. The complete ITS region was amplified using the ITS1f-ITS4 primer pair [41]. PCR-generated amplicons were sequenced on the 454-pyrosequencing platform using the GS-FLX + technology and titanium chemistry as implemented by Beckman Coulter Genomics (Danvers, MA, USA). Raw sequences were processed and filtered using the QIIME pipeline [38]. Authors trimmed reads, excluded reads less than $350 \mathrm{bp}$ and reads containing homopolymers greater than $10 \mathrm{bp}$, denoised reads via flowgram clustering, and filtered reads using the chimera detection and removal algorithm implemented in USEARCH [42]. OTUs were clustered at $97 \%$ sequence similarity using the USEARCH algorithm. Taxonomy was assigned using a custom curated fungal ITS database [43]. Complete sampling, molecular, and bioinformatic methodology used to generate OTU tables can be found in Talbot et al. [11]. The original authors provided the processed OTU and taxonomy tables.

\section{Tedersoo et al. [12]—Global Northern Temperate Soil Fungi}

This data set, includes 131 globally distributed observations of soil fungal community composition within northern temperate latitudes, collected over 1266 days with a standardized sampling methodology using a $5 \mathrm{~cm}$ soil corer, sampling to $5 \mathrm{~cm}$ depth [12]. DNA were extracted using the PowerMax Soil DNA Isolation kit (MoBio, Carlsbad, CA USA). Forward and degenerate reverse primers targeting the ITS2 region were designed to match $>99.5 \%$ of all fungi. PCR-generated amplicons were sequenced on the 454pyrosequencing platform using the GS-FLX + technology and titanium chemistry as implemented by Beckman Coulter. Raw sequences were processed and filtered using mothur 1.32.2 [44]. Sequences, barcodes, and primers were trimmed. Authors excluded reads less than $300 \mathrm{bp}$, and reads containing homopolymers greater than $12 \mathrm{bp}$. The conserved region of ITS was separated from flanking 5.8 and $28 \mathrm{~S}$ regions using ITS extractor software [45]. OTUs were clustered at $98 \%$ sequence similarity using and UCHIME 4.2, which also performs chimera detection and removal [46]. No "denoising" filtering was performed. Taxonomy was assigned using the UNITE fungal taxonomy database [47]. Complete sampling, molecular, and 
bioinformatic methodology used to generate OTU tables can be found in Tedersoo et al. [12]. The original authors provided the processed OTU and taxonomy tables.

\section{Analysis of fungal community similarity}

We used OTU tables provided by each author, and therefore, we analyze each data set separately as they clustered OTUs based on different ribosomal DNA regions and different bioinformatic choices. Each OTU table was proportionally normalized, such that the per OTU sequence count within a sample was divided by the total number of sequences detected in a given sample generating a proportion on the interval $[0,1]$. This proportional normalization has been demonstrated to be sufficiently accurate when using the Bray-Curtis similarity metric [48]. Bray-Curtis similarity was calculated with the vegdist function within the vegan package for $\mathrm{R}$ statistical software [36, 37]. Bray-Curtis values generated from Talbot et al. [11] and Tedersoo et al. [12]. OTU tables were natural log transformed to meet the assumptions of linear regression. To account for zeros in Bray-Curtis similarity, we added the lowest value of community similarity observed within each data set to every observation. Because both data sets differ in the extent of spatial and temporal distances as well as replication, we visualize both to aid direct comparison and interpretation (Supplementary Fig. 2). We also present the absolute number of samples taken in time (Supplementary Fig. 3) and the spatial distribution of sampling (Supplementary Fig. 4).

We used sampling dates to calculate both intra- and interannual temporal distances in days. Spatial distances in kilometers were calculated using reported latitude and longitude, using the distm function within the geosphere package for $\mathrm{R}$ to account for the curvature of the Earth [49]. Inter-annual temporal distances between samples were calculated as days between sampling time points. Intra-annual temporal distances were calculated by determining the seasonal position of each observation. Each observation's seasonal start and end was determined using NASA MEaSUREs Vegetation Index and Phenology NDVI product [50]. We then calculated how many days since the start of the growing season had passed at the time of sampling. This number was divided by the total season length to get a seasonal position on the interval $0-1$. We excluded sites with multiple growing seasons. In addition to spatial and temporal distances, we included environmental covariates in analyses of fungal community similarity at the continental and global scale. Mean annual temperature (MAT) and mean annual precipitation (MAP) were assigned for each site using the WorldClim global data product [51]. Net primary production (NPP) was assigned for each site using the Atlas and the Biosphere global data product [52]. We calculated distance matrices for the following predictors: soil \%C, soil C:N, \% soil moisture at time of sampling, soil $\mathrm{pH}, \mathrm{NPP}, \mathrm{MAT}, \mathrm{MAP}$, and the annual coefficient of variation of MAT and MAP based on monthly means (MAT_CV and MAP_CV, respectively). To determine the relationships between environmental factors and space or time, we regressed temporal distances against environmental predictor distances in each data set (soil \%C, soil C: $\mathrm{N}, \%$ soil moisture at time of sampling, soil $\mathrm{pH}, \mathrm{NPP}, \mathrm{MAT}$, MAP, and the annual coefficient of variation of MAT and MAP based on monthly means (MAT_CV and MAP_CV, respectively)). Some environmental covariates are correlated in our analyses (Supplementary Figs. 6 and 7), and this will affect parameter uncertainty estimates for those predictors [53]. However, because these covariates are not strongly correlated with spatial or temporal distances (Supplementary Figs. 8 and 9), this should not bias our estimates of spatial or temporal effect sizes.

Multiple linear regression models were used to construct each of our three model types: (1) a model that only included spatial and temporal predictors, (2) a model that only included environmental predictors, and (3) a model that included both spatial-temporal and environmental predictors. Intra-annual distance was calculated based on the difference in each observation's seasonal position. Interannual distance was based on the total temporal distance in days between two observations. Models were fit using the MRM function within the ecodist package for $\mathrm{R}$ statistical software [36, 54]. Nonsignificant $(p>0.05)$ predictors were excluded from final models and bootstrap parameter estimate simulations in model types (2) and (3), save for space and time, which are the focus of this analysis.

To better conceptualize the relative importance of spatial and temporal predictors, we calculated how much spatial distance would be required to capture the temporal turnover observed over a 100 day growing season using the parameter estimates from models fit without environmental covariates. Similarly, we calculated how much time would be required to capture the amount of community turnover observed over $100 \mathrm{~km}$ of space.

To test if temporal effects could emerge spuriously due to data structure, analysis approach, or any other confounding factor within our fungal data sets, we performed a Monte Carlo simulation. We repeated our MRM analysis of global northern temperate soil fungi 1000 times, randomizing intra and inter-annual temporal distances. We analyzed the distribution of temporal effect sizes detected in the 1000 simulations and counted how many of these simulations detected a significant effect of time at $p<0.05$. We checked if the distribution of detected effect sizes was approximately normal, if less than $5 \%$ of simulations detect a significant effect at $p<0.05$, and if significant effects detected are equally likely to be positive or negative. 
All code to replicate data manipulation, analysis and figure generation in available at github.com/colinaverill/ Averill_ISMEJ_2019. In addition, we include a custom R function, "space_time_analysis.r", which allows a user to estimate their ability to detect temporal and other effect sizes as they relate to community similarity as done in this manuscript. This function takes a user's particular data set, and returns parameter estimates as well as $95 \%$ parameter confidence intervals. This will allow a user to determine if temporal effects are present in their data set. In the event the temporal parameter confidence interval overlaps zero (temporal effect not significant), the user can determine if some predetermined biologically effect size also falls within the parameter $95 \%$ confidence interval. If this is the case, it likely indicates low statistical power, and the user should not conclude that the temporal effect is not present.

Further use of this function is elaborated within the project repository readme file.

\section{Results}

\section{Can true spatial and temporal effects be detected via analysis of nonorthogonal observations in space and time?}

Analysis of the BCI tropical tree data set and La Selva soil bacterial and fungal data sets, and the Harvard Forest fungal data set revealed significant negative relationships between Bray-Curtis community similarity and both space and time, for both the full and subsetted data sets (Supplementary Fig. 5). Spatial and temporal parameter estimates were not significantly different between full and subsetted analyses based on comparison on 95\% confidence intervals (Supplementary Table 1).

\section{Effects of space and time on soil fungal community similarity}

Models fit to space and time only, without environmental covariates, explained $3.4 \%$ of overall variation in global northern temperate fungal community similarity and $28 \%$ of overall variation in North American soil fungal community similarity, respectively (Fig. 2). We found significant relationships between soil fungal community similarity and space in both the global northern temperate data set $(p<$ 0.01 , Fig. 3a) and the North American fungal data set $(p<$ 0.01 , Fig. $3 d$ ). In addition, we found a significant relationship between fungal community similarity and intra-annual time in the North American fungal data set $(p<0.01$, Fig. 3e). Models demonstrate that soil fungal communities that are closer together in space or time are more similar than those that are farther apart. Using the parameter estimates from our space and time only models, we calculated that 1 season of intra-annual soil fungal community turnover is equivalent to that observed over $\sim 4000 \pm 1100 \mathrm{~km}$ of spatial distance in the North American soil fungal model, fit to spatial and temporal predictors alone. Similarly, $100 \mathrm{~km}$ of spatial turnover in soil fungal community similarity is equivalent to that observed over $6.8 \pm 1.9$ intra-annual days in the North American soil fungal analysis, assuming a growing season length of 265 days, the mean growing season duration the North American data set.

\section{The role of environmental covariates in shaping soil fungal community similarity}

Relationships between fungal community composition and space and time were partly related to how climate and soil resources varied across both space and time. When we fit models to environmental factors only (i.e., without spatiotemporal predictors), we found significant relationships between soil fungal community similarity and soil $\mathrm{pH}$, MAT, and the coefficients of variation for MAP across both datasets (Supplementary Table 1). In addition, soil \%C, NPP, MAP, and the coefficient of variation for MAT were significantly related to fungal community composition in the North American soil fungal dataset $(p<0.01)$. Soil C:N and soil moisture were significantly related to community composition in the global northern temperate data set $(p<$ 0.01 ). These environmental factor-only models, fit without spatial and temporal distances, could explain $44 \%$ of overall variation in North American soil fungal community similarity and $48 \%$ of overall variation in global northern temperate fungal community similarity.

Folding these environmental covariates into spatiotemporal models, we found that all environmental covariates found significant in environment-only models were retained $(p<0.01)$. Models that included space, time, and all environmental covariates could explain $49 \%$ of overall variation in global northern temperate fungal community similarity (Fig. 3a-d) and $46 \%$ of overall variation in North American fungal community similarity ( $p<0.01$, Fig. 2). However, space was no longer significant in predicting fungal community composition in these models of the global northern temperate dataset, but inter-annual time became significant. In models of North American soil fungi, spatial distance, and intra-annual time were retained as a significant predictors of fungal community composition in the space+time+environment model, though effect sizes were lower compared to the model fit with only spatial-temporal covariates. After controlling for environmental covariates, the spatial effect was reduced by $57 \%$ in the North American fungal dataset. In both datasets, spatial 
Fig. 2 Total model fit of spacetime only and space-time plus environment models. 1:1 lines are plotted in black and a smoothing spline is plotted in green. Within the global northern temperate soil fungal data set, space and time alone could explain $3 \%$ of variation (a), and the addition of environmental covariates increased explanatory power to 49\% (b). Within the North American soil fungal data set, space and time alone could explain $28 \%$ of variation (c), and the addition of environmental covariates increased explanatory power to $46 \%$ (d)

\section{Global Northern Temperate Soil Fungi}
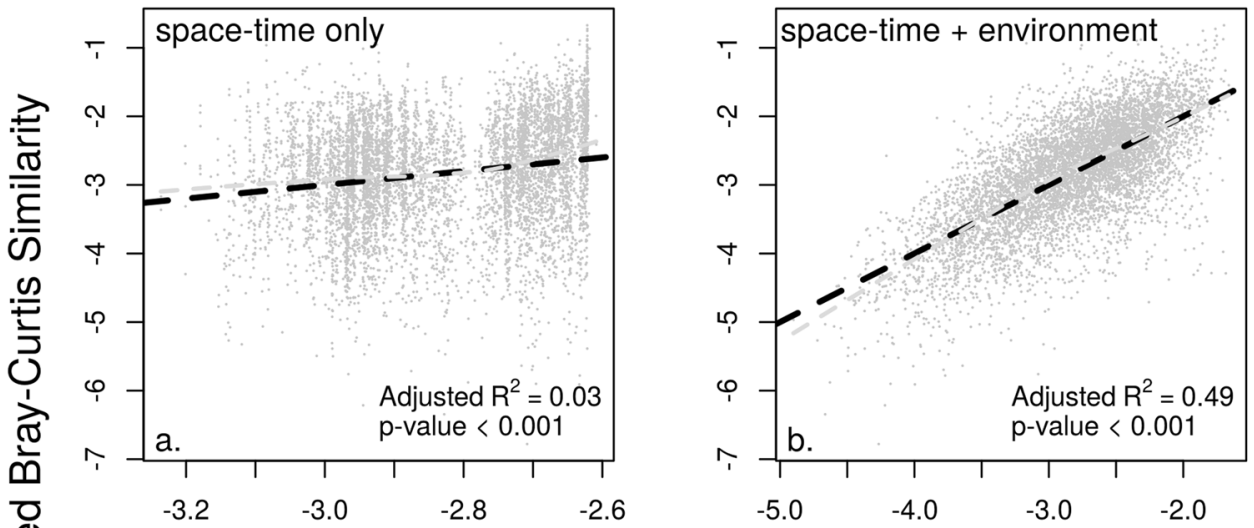

North American Soil Fungi
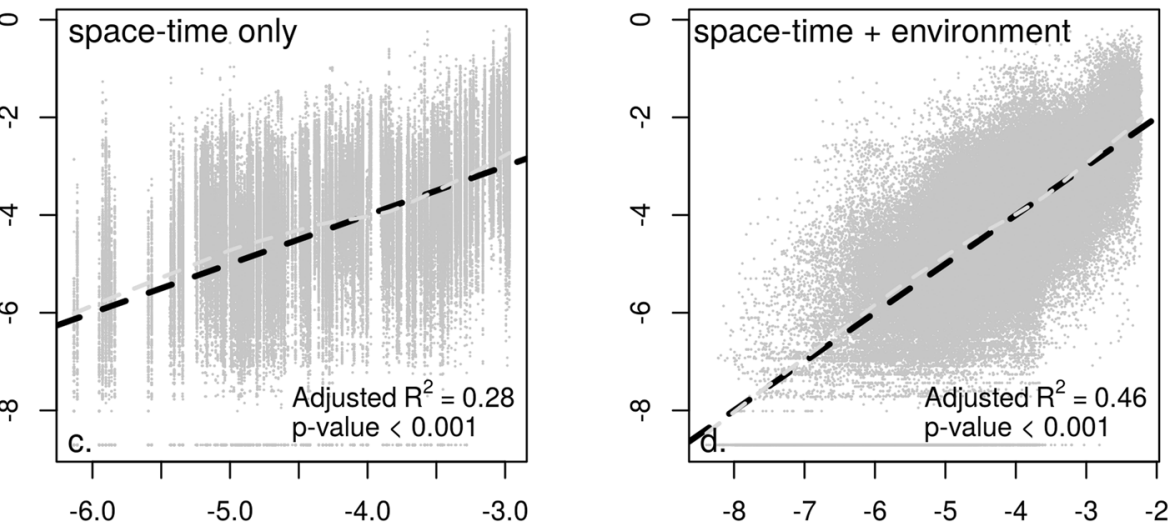

Fitted log-transformed Bray-Curtis Similarity distance between samples was related to changes in MAT and MAP more so than changes in soil resources and soil chemistry (Supplementary Figs. 8 and 9), suggesting that the relationship between fungal community composition and space may be due largely to changes in climate over the sampling sites.

By contrast, sampling time appeared to be an important factor related to fungal community composition in and of itself. In both datasets, temporal predictors were significant in the space+time+environmental factor models, though temporal parameter estimates changed compared to the model fit with only spatial and temporal covariates. Interannual time was significantly related to fungal community composition in the space+time+environment model within the global northern temperate data set $(p<0.01)$, in contrast to models fit to sampling space and time alone. In models of North American soil fungi, after controlling for environmental covariates, the correlation between fungal community composition and intra-annual time decreased by $55 \%$, while the correlation between fungal community composition an inter-annual time remained insignificant. To probe whether soil or climate covariates drove the change in temporal parameter estimates, we fit another set of models. Models fit with models with space, time and climate covariates (MAT, MAP, MAT_CV, and MAP_CV) alone showed that climate could explain most of the decline in the intra-annual effect, and these climate predictors showed stronger correlations with temporal distances than soil factors (Supplementary Figs. 8 and 9).

\section{Monte-Carlo simulation results}

Monte-Carlo simulation of the global northern temperate soil fungi dataset detected a significant $(p<0.05)$ effects of both intra- and inter-annual time on community similarity in $5.5 \%$ and $4.1 \%$ of simulations, respectively, which were equally likely to be positive or negative. Detected effect sizes were normally distributed with a mean of zero (Supplementary Fig. 10). This suggests that detected intra- and inter-annual temporal effects on soil fungal community similarity are not an artifact of data structure. 


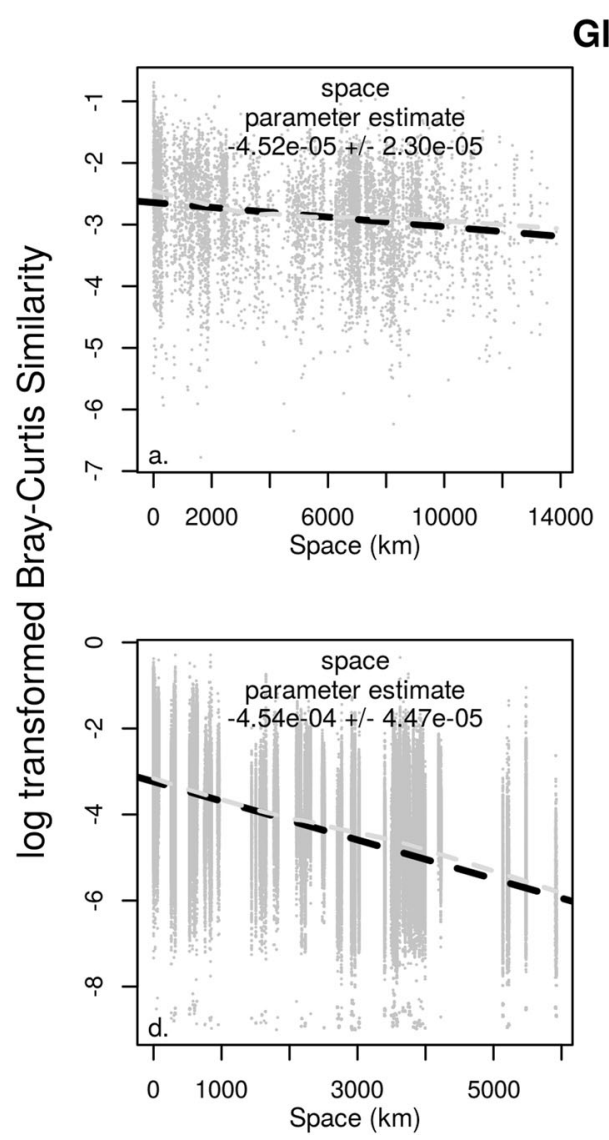

Global Northern Temperate Soil Fungi
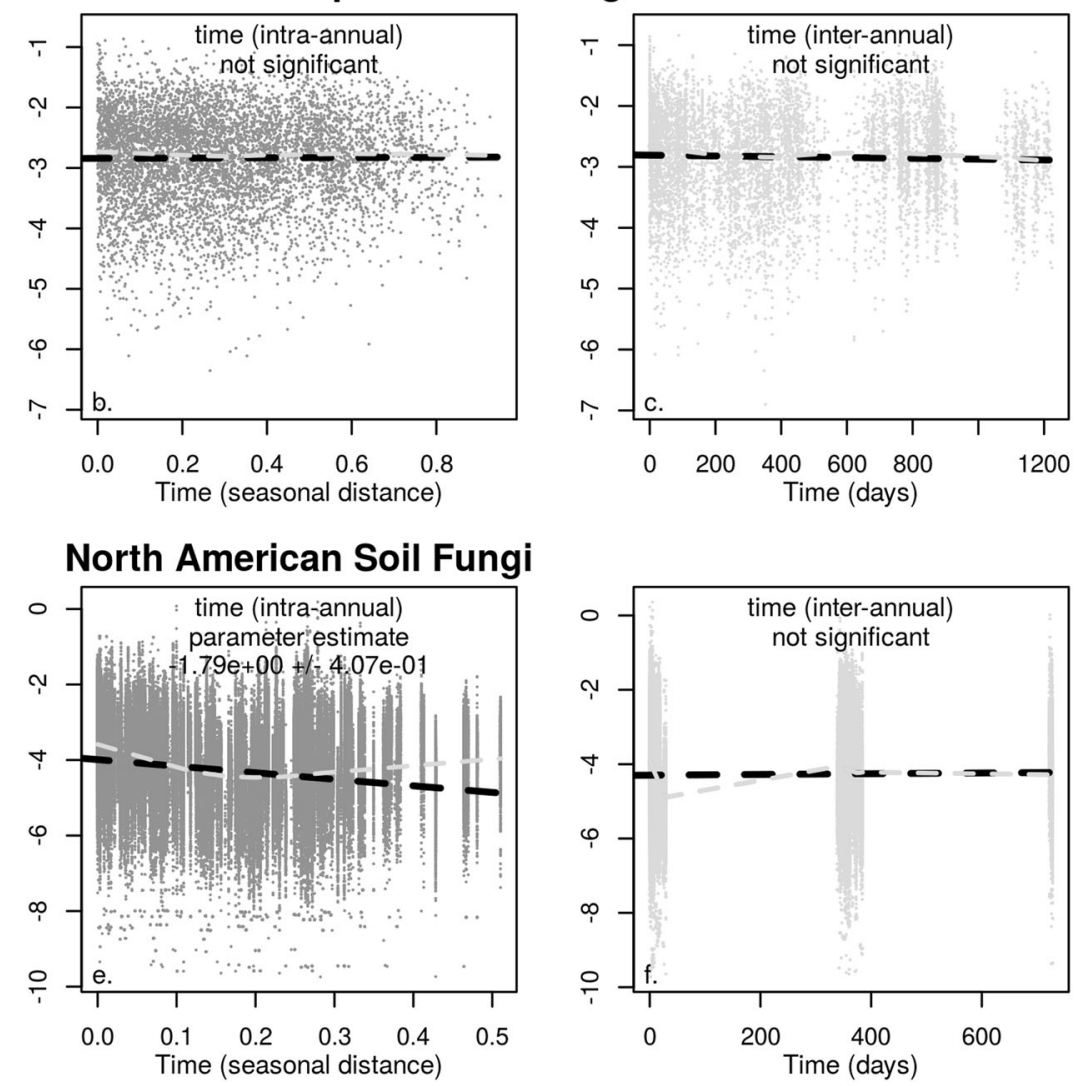

Fig. 3 Effects of space and time on soil fungal community similarity, without accounting for environmental covariates. Ordinary least squares best-fit lines are plotted in black, a smoothing spline is plotted in green. Mean parameter estimates from the full model output are reported along with the $95 \%$ bootstrap confidence interval. We

detected negative effects $(p<0.001)$ of space in both the North American (a) and Global Northern Temperate (b) datasets. We detected a negative effect intra-annual time $(p<0.001)$ in the North American data set

\section{Discussion}

There is increasing evidence that the identity, abundances, and interactions of soil microbial community members translate to variation in microbial community function with ecosystem-scale consequences $[55,56]$. Furthermore, these community level processes are resistant to some degree of environmental change [20,57]. Forecasting the duration of microbial functional legacies requires understanding the rate of microbial community composition turnover in time, which may erode microbial functional legacies. Here, we present the first baseline estimates of soil fungal community turnover rates at large spatial and temporal scales. These numbers can be estimated without repeated temporal measures of soil microbial community composition (Supplementary Fig. 5, Supplementary Table 1). We found support for our hypothesis that temporal changes in fungal community similarity are large (comparable to the turnover observed across thousands kilometers of space), and that inter-annual rates of soil microbial community turnover are comparatively slower than intra-annual rates (Figs. 3 and 4,

Table 1). Changes in fungal community composition with space and time appear to be partly due to changes in certain environmental conditions (i.e., MAT and MAP variation with space), despite the fact that correlations between space, time, and other environmental predictor distances were generally weak (Supplementary Figs. 8 and 9). We also found evidence that some of the variation in fungal community turnover with time cannot be explained by changes in the environment. In fact, controlling for environmental covariates in some cases increased our ability to detect temporal effects. These findings suggest that either additional environmental drivers are important for explaining variation in soil fungal community similarity in both space and time, or that more ecological processes (e.g., dormancy, persistence, and ecological drift) are at play.

We observed intra-annual temporal turnover in soil fungal community composition in the North American soil fungal data set, suggesting that seasonality plays an important role in structuring soil fungal communities. Intra-annual turnover was not detected in the global survey of Northern temperate fungi, and this may reflect the greater 


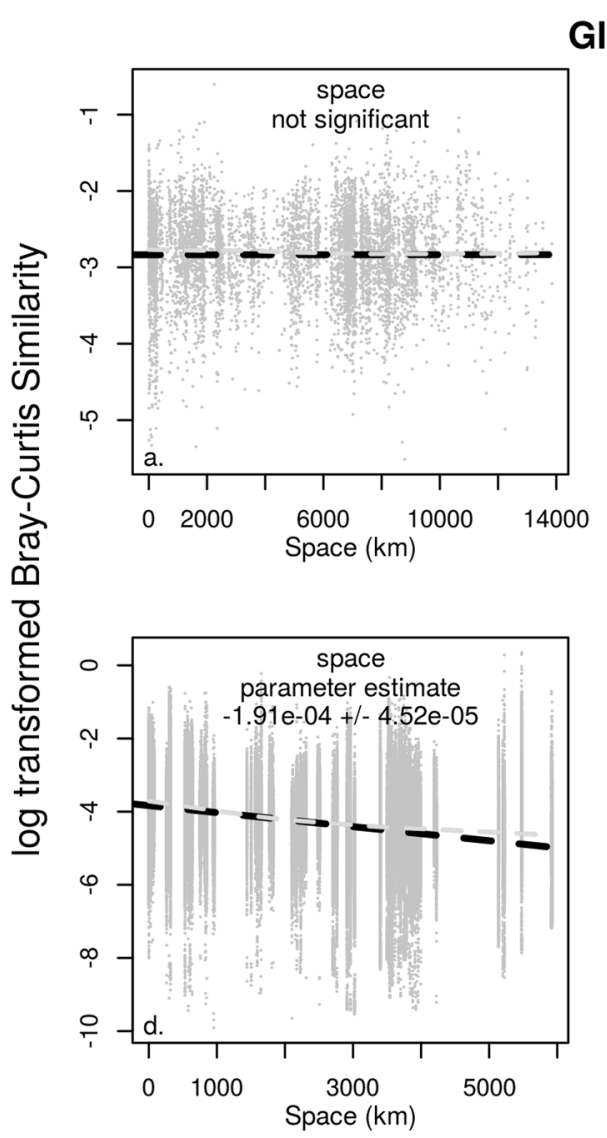

\section{Global Northern Temperate Soil Fungi}
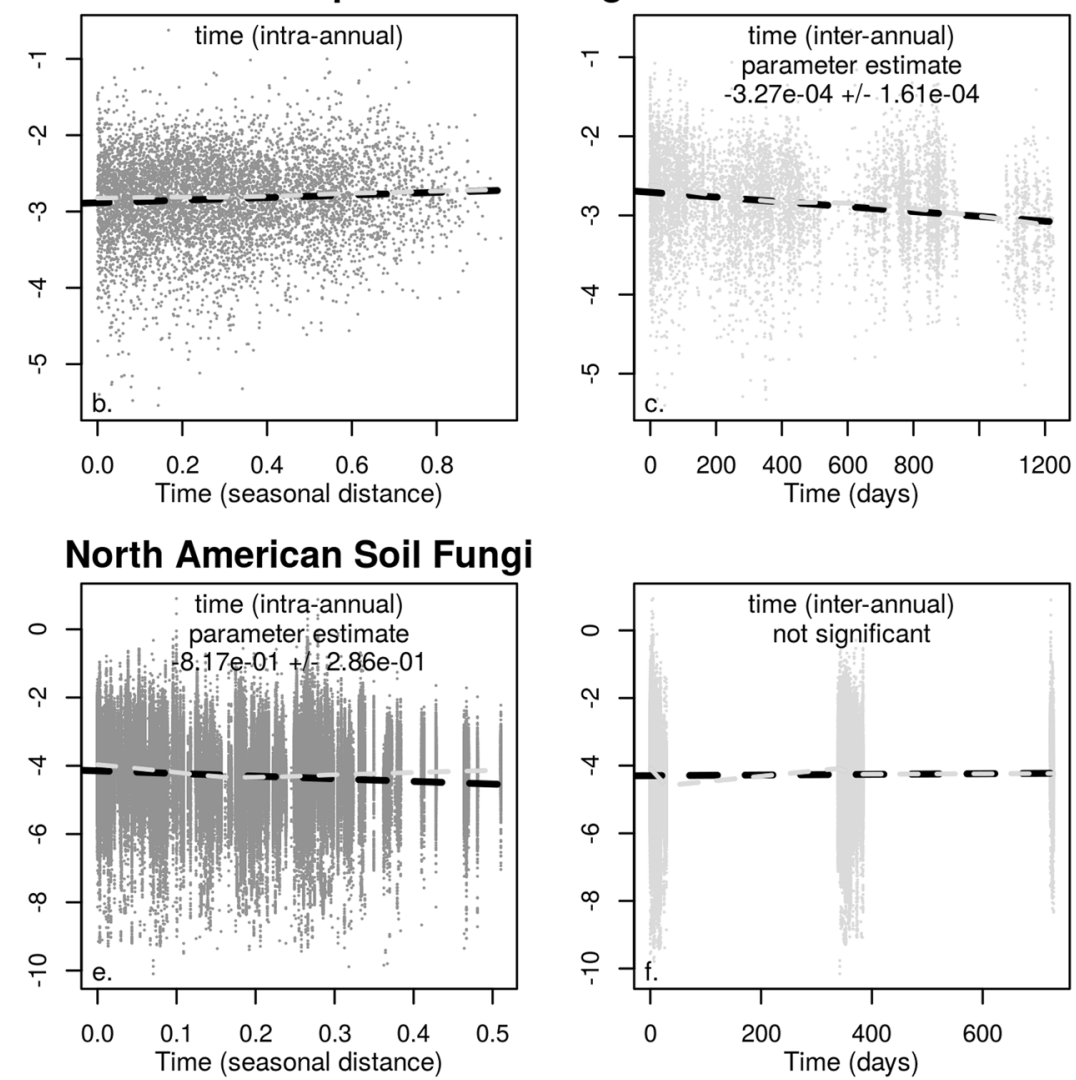

Fig. 4 Effects of space and time on soil fungal community similarity, controlling for environmental covariates. Ordinary least squares bestfit lines are plotted in black, a smoothing spline is plotted in green. Mean parameter estimates from the full model output are reported along with the $95 \%$ bootstrap confidence interval. Global Northern Temperate data were fit as a function of space, intra-annual and interannual time, C:N, \% soil moisture, pH, MAT, MAT coefficient of variation, and MAP coefficient of variation. North American data were fit as a function of space, intra-annual and inter-annual time, \%C, \% soil moisture, $\mathrm{pH}$, MAT, MAP, NPP, MAT coefficient of variation.

Plotted Bray-Curtis community similarity values with space $(\mathbf{b}, \mathbf{f})$ intra-annual time $(\mathbf{c}, \mathbf{g})$, and inter-annual time $(\mathbf{d}, \mathbf{h})$ have been adjusted to control for other covariates in the model and emphasize the variation associated with each predictor in isolation. We detected negative effects $(p<0.001)$ of space in the North American (f), but not the global northern temperate soil fungal data set $(\mathbf{b})$. We detected a negative effect intra-annual time $(p<0.001)$ in both studies. We detected a negative effect of inter-annual time $(p<0.001)$ in the Tedersoo et al. [12] data set (e), but found no effect of time in the Talbot et al. [11] data set (f)

diversity of plant types in the global survey. The North American study focused on plant hosts within the Pinaceae family, while the global northern temperate data set spanned multiple biome types and both hardwood and coniferous plant communities. If Pinaceae dominated ecosystems support a more consistent set of soil fungi, then it may be easier to detect intra-annual turnover among a set of samples where plant communities are more constrained. Given that seasonality is frequently observed in soil fungal communities at the local scale [14], we expect greater sampling effort to reveal stronger intra-annual dynamics at the global scale. By contrast, inter-annual temporal community turnover was observed in the global data set, but not the North American fungal data set. This difference in intra- vs. interannual temporal effects may reflect a difference in statistical power among the studies. The global northern temperate data set [12] represents twice the sampling duration of the
North American soil fungal data set [11], which only included two inter-annual cycles. If North American fungal communities were monitored for a longer duration, we expect a similar effect of inter-annual temporal distance to emerge. Previous analyses of microbial community composition in time have come to a similar conclusion: a lack of temporal effects may merely reflect low sampling effort in many microbial surveys [13].

Consistent with our hypotheses, much of the spatial variation in fungal community composition could be accounted for by shifts in climate. This is distinct from previous continental-scale surveys of soil bacteria that find little effect of climate [10, 18]. Both fungal data sets revealed decay in community similarity in space, yet this was completely explained in the global dataset by the inclusion of environmental covariates. When environmental covariates were added to the model, we could no longer 


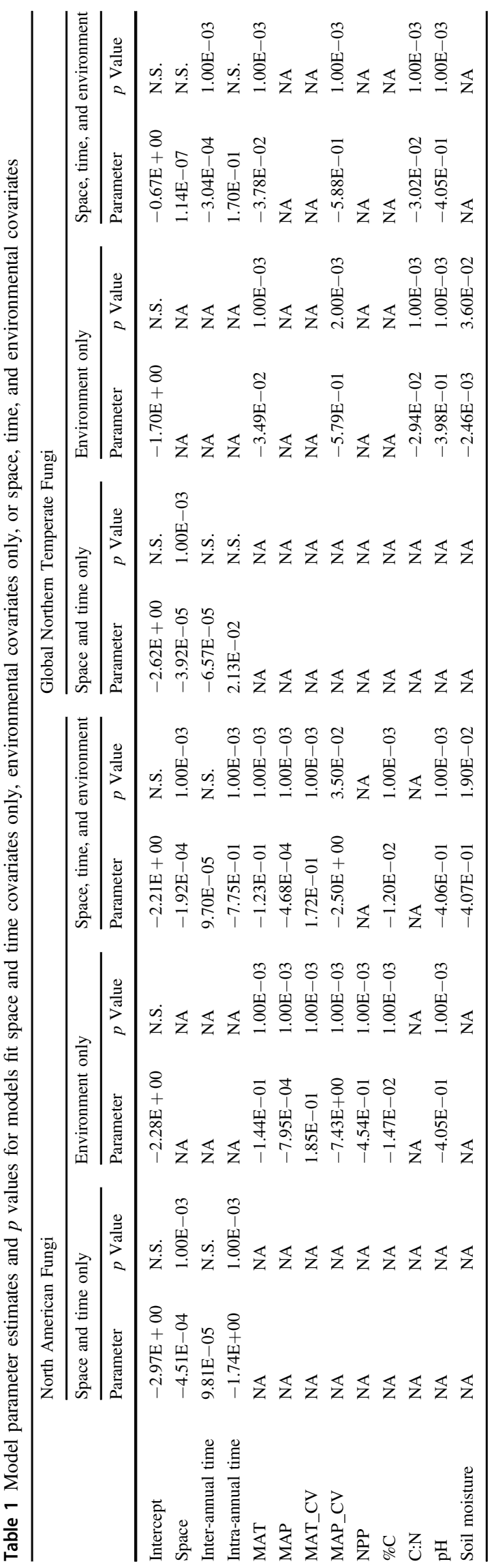

detect a significant effect of space, compared to a model fit to spatial-temporal predictors alone (Fig. 3). This was not true of the North American soil fungal data set, despite the inclusion of the same set of environmental covariates. It may be that larger spatial variation in environmental covariates in the global soil fungal sampling swamped other spatial ecological signatures at finer scales such that they could not be detected, and finer spatial scale sampling in the global dataset would reveal these effects. Past studies of soil fungal community similarity in space at finer scales have revealed patterns consistent with purely ecological factors driving persistent variation in soil fungi in space [58]. This concept is consistent with the fact that the mean spatial distance among sites is over twice as large in the global northern temperate data set as in the North American dataset.

By contrast, our Monte-Carlo analysis of temporal effects in the global northern temperate dataset indicated that the temporal effects detected in this analysis are unlikely due to some other aspect of data structure driving spurious correlations. Some of the temporal variation could be explained by the environmental covariates included in our models, which reduced the magnitude of some temporal parameter estimates in our models (Fig. 3 vs. 4). However, not all of the temporal variation observed could be explained by accounting for these environmental covariates. This suggests two possibilities, which are not mutually exclusive. First, there are likely important environmental drivers that vary within and between years, which are not accounted for in current large-scale soil microbial surveys. This could include seasonal variation in plant belowground $\mathrm{C}$ allocation [59], soil nutrient availability [60], and other environmental and ecological factors that vary in time. Second, ecological processes related to dormancy and persistence traits, as well as neutral ecological and evolutionary processes, can generate temporal effects in fungal community similarity, even if all environmental factors are accounted for. For instance, tradeoffs in ectomycorrhizal colonization vs. competitive ability can drive structure and turnover of ectomycorrhizal communities in space, independent of other environmental controls [5]. Similar purely ecological drivers in time (i.e., predator-prey cycles, variation in dormancy or persistence traits, and ecological drift) could explain why temporal effects persist even if all environmental factors were accounted for.

This study represents the first effort to directly compare the spatial vs. temporal controls over soil fungal community similarity at continental and global scales. These estimates provide a baseline of how long microbial functional legacies may last in the face of ongoing global environmental change, to the extent they are driven by differences in soil fungal community composition. The rate of multidimensional environmental change both within and between 
years-including changes in seasonality, climate, and other anthropogenic forcings-likely varies over our sampling space, and will likely affect the rate of temporal community turnover in a way that we could not capture in this study. We fully acknowledge there are multiple temporal drivers of soil microbial community composition not addressed by our study, especially local-scale heterogeneity in the rate of climate change [24], and likely contribute to uncertainty in our estimates (Fig. 1). Nevertheless, the importance of temporal controls over soil microbial similarity have been either implicitly under-estimated or ignored in the literature at present (but see [13, 14]). Here, we show that temporal controls over the scale of a year are equivalent in magnitude to hundreds to thousands of kilometers of space, yet interannual rates of turnover are comparatively slow, pointing to potentially long-lasting microbial community legacies.

Acknowledgements Colin Averill was supported by the NOAA Climate and Global Change Postdoctoral Fellowship Program, administered by Cooperative Programs for the Advancement of Earth System Science (CPAESS), University Corporation for Atmospheric Research (UCAR), Boulder, Colorado, USA, as well as NSF Macrosystems Biology \#1638577. Michael Dietze was supported by NSF Macrosystems Biology \#1318164 and \#1638577. LeAnna Cates was supported by the Boston University Bioinformatics BRITE Research Experiences for Undergraduates Program. Jennifer Bhatnagar was supported by NSF Macrosystems Biology \#1638577.

\section{Compliance with ethical standards}

Conflict of interest The authors declare that they have no conflict of interest.

Publisher's note: Springer Nature remains neutral with regard to jurisdictional claims in published maps and institutional affiliations.

\section{References}

1. van der Heijden MGA, Martin FM, Selosse M-A, Sanders IR. Mycorrhizal ecology and evolution: the past, the present, and the future. New Phytologist. 2015;205:1406-23.

2. Leibold MA. The niche concept revisited: mechanistic models and community context. Ecology. 1995;76:1371-82.

3. Hubbell SP. The unified neutral theory of biodiversity and biogeography. Princeton: Princeton University Press; 2001.

4. Peay KG, Garbelotto M, Bruns TD. Evidence of dispersal limitation in soil microorganisms: isolation reduces species richness on mycorrhizal tree islands. Ecology. 2010;91:3631-40.

5. Smith GR, Steidinger BS, Bruns TD, Peay KG. Competitioncolonization tradeoffs structure fungal diversity. ISME J 2018;12: $1758-67$.

6. Jones SE, Lennon JT. Dormancy contributes to the maintenance of microbial diversity. Proc. Natl Acad. Sci. 2010;107:5881-6.

7. Hanson CA, Allison SD, Bradford MA, Wallenstein MD, Treseder KK. Fungal taxa target different carbon sources in forest soil. Ecosystems. 2008;11:1157-67.

8. McGuire KL, Bent E, Borneman J, Majumder A, Allison SD, Tresederi KK. Functional diversity in resource use by fungi. Ecology. 2010;91:2324-32.
9. Kaiser C, Koranda M, Kitzler B, Fuchslueger L, Schnecker J, Schweiger P, et al. Belowground carbon allocation by trees drives seasonal patterns of extracellular enzyme activities by altering microbial community composition in a beech forest soil. New Phytologist. 2010;187:843-58.

10. Lauber CL, Hamady M, Knight R, Fierer N. Pyrosequencingbased assessment of soil $\mathrm{pH}$ as a predictor of soil bacterial community structure at the continental scale. Appl. Environ. Microbiol. 2009;75:5111-20.

11. Talbot JM, Bruns TD, Taylor JW, Smith DP, Branco S, Glassman SI, et al. Endemism and functional convergence across the North American soil mycobiome. Proc. Natl Acad. Sci. 2014;111: 6341-6.

12. Tedersoo L, Bahram M, Polme S, Koljalg U, Yorou NS, Wijesundera R, et al. Global diversity and geography of soil fungi. Science. 2014;346:1256688-1256688.

13. Shade A, Gregory Caporaso J, Handelsman J, Knight R, Fierer N. A meta-analysis of changes in bacterial and archaeal communities with time. ISME J. 2013;7:1493-506.

14. Bahram M, Peay KG, Tedersoo L. Local-scale biogeography and spatiotemporal variability in communities of mycorrhizal fungi. New Phytologist. 2015;205:1454-63.

15. Voříšková J, Brabcová V, Cajthaml T, Baldrian P. Seasonal dynamics of fungal communities in a temperate oak forest soil. New Phytologist. 2014;201:269-78.

16. Franklin RB, Mills AL. Multi-scale variation in spatial heterogeneity for microbial community structure in an eastern Virginia agricultural field. FEMS Microbiol. Ecol. 2003;44:335-46.

17. Lauber CL, Ramirez KS, Aanderud Z, Lennon J, Fierer N. Temporal variability in soil microbial communities across landuse types. ISME J. 2013;7:1641-50.

18. Fierer N, Jackson RB. The diversity and biogeography of soil bacterial communities. Proc. Natl Acad. Sci. 2006;103:626-31.

19. Hawkes CV, Keitt TH. Resilience vs. historical contingency in microbial responses to environmental change. Ecol. Lett. 2015; 18:612-25.

20. Allison SD, Martiny JBH. Resistance, resilience, and redundancy in microbial communities. Proc. Natl Acad. Sci. 2008;105: 11512-9.

21. Bradford MA, Davies CA, Frey SD, Maddox TR, Melillo JM, Mohan JE, et al. Thermal adaptation of soil microbial respiration to elevated temperature. Ecol. Lett. 2008;11:1316-27.

22. Evans SE, Wallenstein MD. Soil microbial community response to drying and rewetting stress: does historical precipitation regime matter? Biogeochemistry. 2012;109:101-16.

23. Averill C, Waring BG, Hawkes CV. Historical precipitation predictably alters the shape and magnitude of microbial functional response to soil moisture. Glob. Change Biol. 2016;22:1957-64.

24. Dobrowski SZ, Abatzoglou J, Swanson AK, Greenberg JA, Mynsberge AR, Holden ZA, et al. The climate velocity of the contiguous United States during the 20th century. Glob. Change Biol. 2013;19:241-51.

25. Bray JR, Curtis JT. An ordination of the upland forest communities of Southern Wisconsin. Ecol. Monogr. 1957;27:325-49.

26. Anderson MJ, Crist TO, Chase JM, Vellend M, Inouye BD, Freestone AL, et al. Navigating the multiple meanings of $\beta$ diversity: a roadmap for the practicing ecologist: Roadmap for beta diversity. Ecol. Lett. 2011;14:19-28.

27. Condit R. Tropical forest census plots: methods and results from Barro Colorado Island, Panama, and a comparison with other plots. Berlin; New York, NY: Springer; 1998.

28. Hubbell SP. Light-gap disturbances, recruitment limitation, and tree diversity in a neotropical forest. Science. 1999;283:554-7.

29. Hubbell SP, Condit R, Foster RB. Barro Colorado Forest Census Plot Data. 2005. 
30. Kivlin SN, Hawkes CV. Temporal and Spatial Variation of Soil Bacteria Richness, Composition, and Function in a Neotropical Rainforest. PLOS ONE. 2016;11:e0159131.

31. Kivlin SN, Hawkes CV. Tree species, spatial heterogeneity, and seasonality drive soil fungal abundance, richness, and composition in Neotropical rainforests: Soil fungi in Neotropical rainforest. Environ. Microbiol. 2016;18:4662-73.

32. Eugenio González J, Fisher RF. Growth of native forest species planted on abandoned pasture land in Costa Rica. Forest Ecol Manag. 1994;70:159-67.

33. Dickie IA. Insidious effects of sequencing errors on perceived diversity in molecular surveys: letters. New Phytologist. 2010; 188:916-8.

34. Wang Q, Garrity GM, Tiedje JM, Cole JR. Naive Bayesian Classifier for rapid assignment of rRNA sequences into the new bacterial taxonomy. Appl. Environ. Microbiol. 2007;73:5261-7.

35. Love MI, Huber W, Anders S. Moderated estimation of fold change and dispersion for RNA-seq data with DESeq2. Genome Biol. 2014;15:550.

36. $\mathrm{R}$ Core Team. R: A language and environment for statistical computing. Vienna, Austria: R Foundation for Statistical Computing; 2017.

37. Oksanen J, Blanchet GF, Kindt R, Legendre P, Minchin PR, O'Hara RB, et al. vegan: Community Ecology Package. 2015.

38. Caporaso JG, Kuczynski J, Stombaugh J, Bittinger K, Bushman FD, Costello EK, et al. QIIME allows analysis of high-throughput community sequencing data. Nat. Methods. 2010;7:335-6.

39. Callahan BJ, McMurdie PJ, Holmes SP. Exact sequence variants should replace operational taxonomic units in marker-gene data analysis. ISME J. 2017;11:2639-43.

40. Callahan BJ, McMurdie PJ, Rosen MJ, Han AW, Johnson AJA, Holmes SP. DADA2: high-resolution sample inference from Illumina amplicon data. Nat. Methods. 2016;13:581-3.

41. White TJ, Bruns T, Lee S, Taylor JW. Amplification and direct sequencing of fungal ribosomal RNA genes for phylogenetics. PCR Protocols: A Guide to Methods and Applications. New York, NY: Academic Press, Inc.; 1990. p. 315-22.

42. Edgar RC. Search and clustering orders of magnitude faster than BLAST. Bioinformatics. 2010;26:2460-61.

43. Peay KG, Baraloto C, Fine PVA. Strong coupling of plant and fungal community structure across western Amazonian rainforests. ISME J. 2013;7:1852-61.

44. Schloss PD, Westcott SL, Ryabin T, Hall JR, Hartmann M, Hollister EB, et al. Introducing mothur: open-source, platformindependent, community-supported software for describing and comparing microbial communities. Appl. Environ. Microbiol. 2009;75:7537-41.

45. Bengtson P, Barker J, Grayston SJ. Evidence of a strong coupling between root exudation, $\mathrm{C}$ and $\mathrm{N}$ availability, and stimulated
SOM decomposition caused by rhizosphere priming effects. Ecol. Evolut. 2012;2:1843-52.

46. Edgar RC, Haas BJ, Clemente JC, Quince C, Knight R. UCHIME improves sensitivity and speed of chimera detection. Bioinformatics. 2011;27:2194-2200.

47. Kõljalg U, Nilsson RH, Abarenkov K, Tedersoo L, Taylor AFS, Bahram M, et al. Towards a unified paradigm for sequence-based identification of fungi. Mol. Ecol. 2013;22:5271-7.

48. Weiss S, Xu ZZ, Peddada S, Amir A, Bittinger K, Gonzalez A, et al. Normalization and microbial differential abundance strategies depend upon data characteristics. Microbiome 2017;5:27.

49. Hijmans RJ, Willians E, Vennes C. geosphere: spherical trigonometry. 2016

50. K. Didan AB. NASA MEaSUREs Vegetation Index and Phenology (VIP) Phenology EVI2 Yearly Global 0.05Deg CMG. 2016. NASA EOSDIS Land Processes DAAC.

51. Hijmans RJ, Cameron SE, Parra JL, Jones PG, Jarvis A. Very high resolution interpolated climate surfaces for global land areas. Int. J. Climatol. 2005;25:1965-78.

52. Foley JA, Prentice IC, Ramankutty N, Levis S, Pollard D, Sitch S, et al. An integrated biosphere model of land surface processes, terrestrial carbon balance, and vegetation dynamics. Glob. Biogeochem. Cycles. 1996;10:603-28.

53. Zuur AF, Ieno EN, Elphick CS. A protocol for data exploration to avoid common statistical problems: data exploration. Methods Ecol. Evolut. 2010;1:3-14.

54. Goslee SC, Urban DL. The ecodist package for dissimilaritybased analysis of ecological data. J. Stat. Softw. 2007;22. https://www.jstatsoft.org/article/view/v022i07.

55. Averill C, Rousk J, Hawkes C. Microbial-mediated redistribution of ecosystem nitrogen cycling can delay progressive nitrogen limitation. Biogeochemistry. 2015;126:11-23.

56. Kyaschenko J, Clemmensen KE, Hagenbo A, Karltun E, Lindahl BD. Shift in fungal communities and associated enzyme activities along an age gradient of managed Pinus sylvestris stands. ISME J. 2017;11:863-74.

57. Hawkes CV, Waring BG, Rocca JD, Kivlin SN. Historical climate controls soil respiration responses to current soil moisture. Proc. Natl Acad. Sci. 2017;114:6322-7.

58. Peay KG, Bruns TD, Kennedy PG, Bergemann SE, Garbelotto M. A strong species-area relationship for eukaryotic soil microbes: island size matters for ectomycorrhizal fungi. Ecol. Lett. 2007; 10:470-80.

59. Högberg P, Nordgren A, Buchmann N, Taylor AFS, Ekblad A, Högberg MN, et al. Largescale forest girdling shows that current photosynthesis drives soil respiration. Nature. 2001;411:789-92.

60. Brzostek ER, Finzi AC. Substrate supply, fine roots, and temperature control proteolytic enzyme activity in temperate forest soils. Ecology. 2011;92:892-902. 